\section{SHOULD WE TAKE MAJOR MACRO-ECONOMIC AND POLITICAL DEVELOPMENTS INTO ACCOUNT WHEN ASSESSING LONG-TERM OCCUPATIONAL EXPOSURES FOR EPIDEMIOLOGICAL RESEARCH?}

${ }^{1}$ Hans Kromhout, ${ }^{2}$ Remko Houba, ${ }^{3}$ Susan Peters, ${ }^{4}$ Joachim Schüz, ${ }^{4}$ Kurt Straif, ${ }^{5}$ Sergey Kashansky, ${ }^{6}$ Evgeny Kovalevskiy, ${ }^{4}$ Sara Schonfeld, ${ }^{4}$ Valerie McCormack, ${ }^{1}$ Roel Vermeulen. ${ }^{1}$ Utrecht University, Utrecht, The Netherlands; ${ }^{2}$ Netherlands Expertise Centre for Occupational Respiratory Disorders, Utrecht, The Netherlands; ${ }^{3}$ University of Western Australia, Perth, Australia; ${ }^{4}$ International Agency for Research on Cancer, Lyon, France; ${ }^{5}$ Medical Research Center for Prophylaxis and Health Protection in Industrial Workers, Yekaterinburg, Russia; ${ }^{6}$ Russian Academy of Medical Sciences, Moscow, Russia

10.1136/oemed-2014-102362.148

Objectives Recent analyses of long-term trends in respirable dust and quartz concentrations from the long term monitoring program of the European Industrial Minerals Association (IMAEurope) Dust Monitoring Program (covering the years 20002013) showed striking downward temporal trends in exposure which came to a halt at around the year 2009. Careful analyses and discussion with occupational health and safety representatives pointed at a direct detrimental effect of the current economic crisis on measured concentrations. This observation led us to hypothesise that similar disruptions of downward temporal trends in occupational exposures might also be visible in other large databases with longitudinal exposure measurements.

Method Temporal time trends were estimated in two additional databases (ExpoSYN and URALASBEST) each covering more than 50 years of occupational exposure monitoring. More flexible spline analyses rather than standard log linear (multiplicative) models were used to look for reversed trends.

Results In all three databases macro-economic and political developments seemed to influence downward trends in occupational exposure concentrations. Effects of economic crises like those of the early 1980s, early 1990s and the most recent one as well as the period of political and economic reform in Russia were clearly visible as reduced downward or even reversed temporal trends in occupational exposure concentrations.

Conclusions In exposure assessment for occupational epidemiological studies long term exposures are often modelled as log linear trends. Approaches allowing for disruptions of these trends by macro-economic and/or political developments are needed for more accurate and precise estimations of long-term exposure and will result in more reliable quantitative risk estimates.

\section{CANJEM: A GENERAL POPULATION JOB EXPOSURE MATRIX BASED ON PAST EXPERT ASSESSMENTS OF EXPOSURE TO OVER 250 AGENTS}

'Jerome Lavoue, ${ }^{2}$ France Labrèche, 'Lesley Richardson, ${ }^{3}$ Mark Goldberg, ${ }^{4}$ Marie-Élise Parent, ${ }^{1,2}$ Jack Siemiatycki. 'University of Montreal Hospital Research Center (CRCHUM), Montreal, Canada; ${ }^{2}$ University of Montreal, Montreal, Canada; ${ }^{3}$ McGill University, Montreal, Canada; ${ }^{4}$ INRS-Institut Armand Frappier, Montreal, Canada

\subsection{6/oemed-2014-102362.149}

Objectives To create a general population job-exposure matrix (JEM) from a database of expert assessments performed during four community-based case-control studies of cancer (lung, breast, brain, and multisite) conducted in Montreal since the 1980s.

Method The expert assessments were performed by the same team of chemists, who assigned exposure to a predefined list of agents to each job held by subjects based on job histories and descriptions of tasks and work environment obtained through interview. The estimated metrics include measures of intensity, frequency and likelihood of exposure. The JEM dimensions include agent, occupation (Canadian, U.S., and international classifications) and era. For each cell, probability of exposure was computed as the proportion of individual jobs exposed. Among the exposed within a cell, CANJEM provides median frequency of exposure, the mode of exposure intensity categories and median time weighted intensity.

Results CANJEM includes information from 6222 men and 2563 women, totalling 31780 individual jobs held between 1921 and 2005, representing approximately 50 expert-years of exposure assessment. Well known agents among the most frequently encountered include carbon monoxide (22\% of individual jobs exposed), organic solvents (17.5\%), and formaldehyde (10.6\%). The JEM covers 303 occupations, 280 agents, and 4 eras $(<1950,1950-1969,1970-1984,>1984)$. Overall, $20 \%$ of the cells have a non-null proportion of jobs exposed, $12 \%$ with a proportion greater than $5 \%$.

Conclusions CANJEM constitutes one of the largest current sources of retrospective occupational exposure information in North America, useable to support exposure assessment efforts in epidemiology and estimate populations of workers exposed to harmful agents.

\section{USE OF AN 0*NET BASED JOB EXPOSURE MATRIX TO PREDICT PREVALENCE OF CARPAL TUNNEL SYNDROME IN A LARGE POOLED COHORT}

${ }^{1}$ Ann Marie Dale, ${ }^{1}$ Angelique Zeringue, ${ }^{2}$ Carisa Harris-Adamson, ${ }^{3}$ Matt Thiese, ${ }^{4}$ Stephen Bao, ${ }^{5}$ Sue Burt, ${ }^{6}$ Linda Merlino, ${ }^{7}$ Arun Garg, ${ }^{2}$ Ellen Eisen, ${ }^{6}$ Fred Gerr, ${ }^{3}$ Kurt Hegmann, ${ }^{7}$ Jay Kapellusch, ${ }^{8}$ David Rempel, ${ }^{4}$ Barbara Silverstein, ${ }^{1}$ Bradley Evanoff. ${ }^{1}$ Washington University School of Medicine, St. Louis, MO, USA; ${ }^{2}$ University of California, Berkeley, Berkeley, CA, USA; ${ }^{3}$ University of Utah, Salt Lake City, UT, USA; ${ }^{4}$ Washington State Department of Labor and Industries, Olympia, WA, USA; ${ }^{5}$ Eformerly National Institute for Occupational Safety and Health, Cincinnati, OH, USA; ${ }^{6} \mathrm{~F}$ University of lowa, lowa City, 10, USA; 'University of Wisconsin-Milwaukee, Milwaukee, WI, USA; ${ }^{8}$ University of California, San Francisco, San Francisco, CA, USA

\subsection{6/oemed-2014-102362.150}

Objectives To determine if job title based physical exposure measures predicted prevalent carpal tunnel syndrome (CTS) in a large pooled cohort of workers.

Method We pooled baseline examination data from six prospective cohort studies, restricting analyses to those employed at least 1 year. CTS was defined as median neuropathy plus typical symptoms. Physical exposure estimates for static strength, dynamic strength, time spent making repetitive motions, and time handling objects were extracted from the Occupational Network (O*NET) database using Standard Occupational Classification codes based on reported job title. Three exposure categories of high force/ high repetition, low force/ low repetition, and mixed high and low exposures were entered into logistic regression models adjusting for age, gender, body mass index (BMI), diabetes, rheumatoid arthritis, employed time and study site.

Results Of 3562 in the pooled cohort, 7.6\% met a prevalent CTS definition with mean employed time of 7.9 years (SD 8.2). Compared to subjects with low job requirements for dynamic strength and repetitive motion, those with mixed exposures or high exposures showed increased prevalence of CTS (OR 1.46; 95\% CI: 1.01-2.11 and OR 2.32; 95\% CI: $1.15-4.67$, respectively). Similar dose dependent associations of combined exposures were shown for all exposure combinations tested, with high/high 